\title{
A Current-Mode TransImpedance Amplifier for Capacitive Sensors ${ }^{+}$
}

\author{
Alessandro Nastro ${ }^{1, *}$, Andrea De Marcellis ${ }^{2}$, Marco Ferrari ${ }^{1}$ and Vittorio Ferrari ${ }^{1}$ \\ 1 Department of Information Engineering, University of Brescia, 25123 Brescia, Italy; \\ marco.ferrari@unibs.it (M.F.); vittorio.ferrari@unibs.it (V.F.) \\ 2 Department of Industrial and Information Engineering and Economics, University of L'Aquila, \\ 67100 L'Aquila, Italy; andrea.demarcellis@univaq.it \\ * Correspondence: a.nastro002@unibs.it; Tel.: +39-030-371-5938 \\ † Presented at the Eurosensors 2018 Conference, Graz, Austria, 9-12 September 2018.
}

Published: 13 November 2018

\begin{abstract}
A Current-Mode (CM) TransImpedance Amplifier (TIA) based on Second Generation Current Conveyors (CCIIs) for capacitive microsensor measurements is presented. The designed electronic interface performs a capacitance-to-voltage conversion using 3 CCIIs and 3 resistors exploiting a synchronous-demodulation technique to improve the overall detection sensitivity and resolution of the system. A CM-TIA solution designed at transistor level in AMS0.35 $\mu \mathrm{m}$ integrated CMOS technology with a power consumption lower than $900 \mu \mathrm{W}$ is proposed. Experimental results obtained with a board-level prototype show linear behavior of the proposed interface circuit with a resolution up to $34.5 \mathrm{fF}$ and a sensitivity up to $223 \mathrm{mV} / \mathrm{nF}$, confirming the theoretical expectations.
\end{abstract}

Keywords: transimpedance amplifier; current conveyor; capacitive sensors; MEMS

\section{Introduction}

Capacitive sensors can be used in a wide range of applications for the detection of mechanical [1,2], chemical $[3,4]$ and physical $[5,6]$ quantities by measuring capacitance variations. Capacitive sensing has gained a crucial role particularly in Micro Electro-Mechanical Systems (MEMS) where quantities such as acceleration, displacement or pressure have to be measured without generating excessive electrostatic forces on the capacitive sensing element [7-10]. A TransImpedance Amplifier (TIA) based on Second Generation Current Conveyors (CCII) with good performances in terms of linearity, sensitivity, resolution and capability to reveal large dynamic ranges of capacitance sensor variations is proposed. In particular, with respect to voltage-mode architectures based on Operational Amplifiers (OAs), CCII-based Current-Mode (CM) solutions are mainly suitable for their integration in a standard CMOS technology through simple architectures with a reduced number of transistors, within a small silicon area and having low-power characteristics [11-13]. The use of CCII promotes the design of integrated circuits for portable sensor applications, overcoming constraints typically provided by circuits based on OAs, ensuring a larger frequency bandwidth and a good capability to work at low supply voltage levels with a wide input-output dynamic range [14,15]. The proposed CM-TIA description and operating principle are illustrated in Section 2, experimental results are presented in Section 3, and conclusions are given in Section 4.

\section{Current-Mode Transimpedance Amplifier}

The block diagram of the CM-TIA is shown in Figure 1a while Figure $1 \mathrm{~b}$ reports the schematic circuit of the proposed CCII designed at transistor level in AMS0.35 $\mu \mathrm{m}$ CMOS integrated technology with a power consumption lower than $900 \mu \mathrm{W}$ at $\pm 1 \mathrm{~V}$ dual supply voltage. Time and frequency 
domain simulation results of the integrated CM-TIA are reported in Figure 2. The current $i_{s}(\mathrm{t})$ produced by the excitation sinusoidal signal $v_{\mathrm{s}}(\mathrm{t})$ applied to the capacitance under test $C_{\mathrm{x}}$ is applied to the CCII low-impedance input X. The three resistors $R_{1}=100 \mathrm{k} \Omega, R_{2}=51 \mathrm{k} \Omega$ and $R_{3}=100 \mathrm{k} \Omega$ set the total gain of the TIA:

$$
\mathrm{G}=v_{\mathrm{t}}(\mathrm{t}) / i_{\mathrm{s}}(\mathrm{t})=\left(2 R_{1} R_{3}\right) / R_{2}
$$

Thanks to the virtual ground at the $\mathrm{CCII}_{1} \mathrm{X}$ node, the current $i_{\mathrm{s}}(\mathrm{t})$ is given by:

$$
i_{s}(\mathrm{t})=2 \pi f_{\mathrm{s}} C_{x} v_{\mathrm{s}}(\mathrm{t})
$$

The output voltage of the TIA $v_{\mathrm{t}}(\mathrm{t})$ is then equal to:

$$
v_{\mathrm{t}}(\mathrm{t})=\mathrm{G} 2 \pi f_{\mathrm{s}} C_{\mathrm{x}} v_{\mathrm{s}}(\mathrm{t})
$$

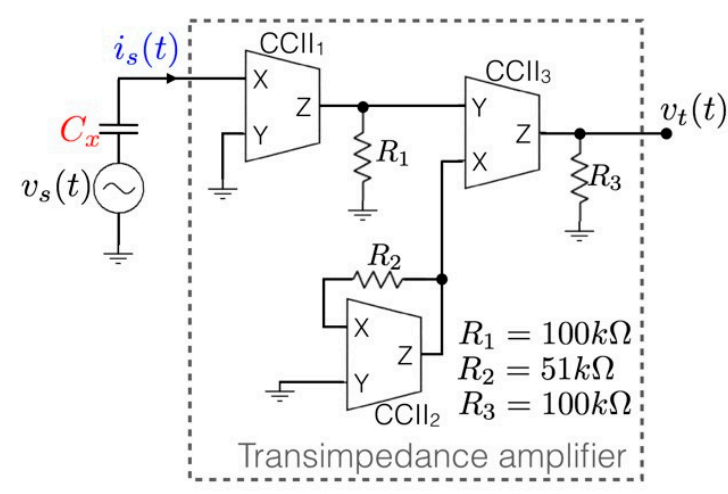

(a)

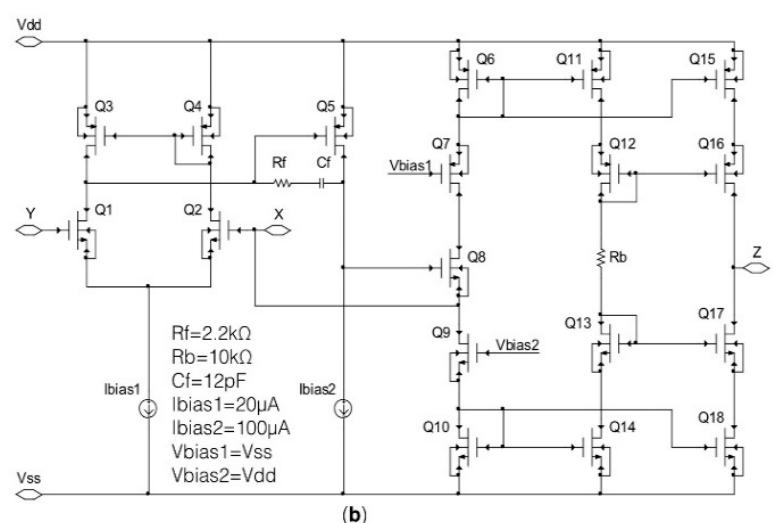

Figure 1. (a) Block diagram of the CCII-based transimpedance amplifier. (b) Transistor-level CCII schematic circuit.

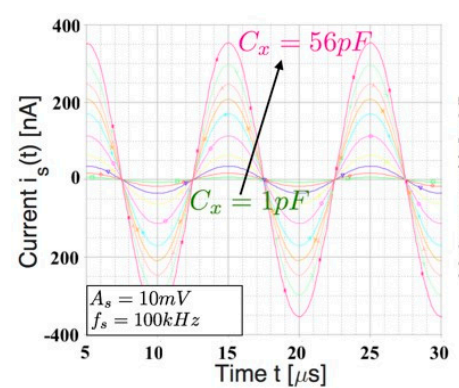

(a)

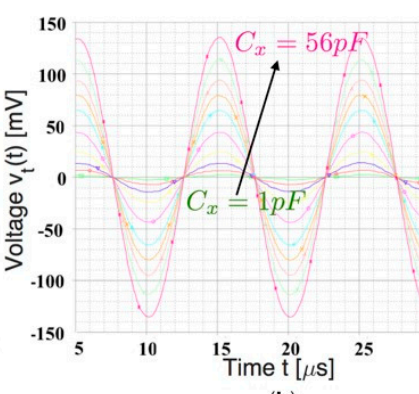

(b)

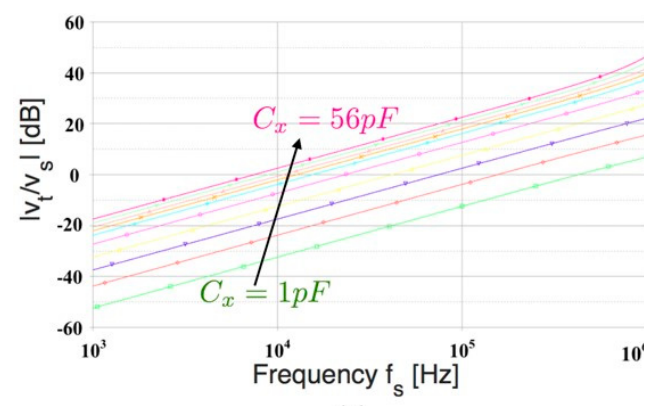

(c)

Figure 2. Time domain simulation results showing (a) the input signals $i_{s}(\mathrm{t})$ and $(\mathbf{b}) v_{s}(\mathbf{t})$ for different capacitance values $C_{x}$. The resulting transimpedance gain $\mathrm{G}$ is about $390 \mathrm{k} \Omega$. (c) Frequency simulation.

\section{Experimental Results}

A board-level prototype of the TIA has been implemented with three commercial CCII (AD844) for preliminary measurements as shown in Figure 3. The experimental frequency responses of the board-level CM-TIA obtained using different reference capacitances within the range of $1-56 \mathrm{pF}$ are reported in Figure 4. 


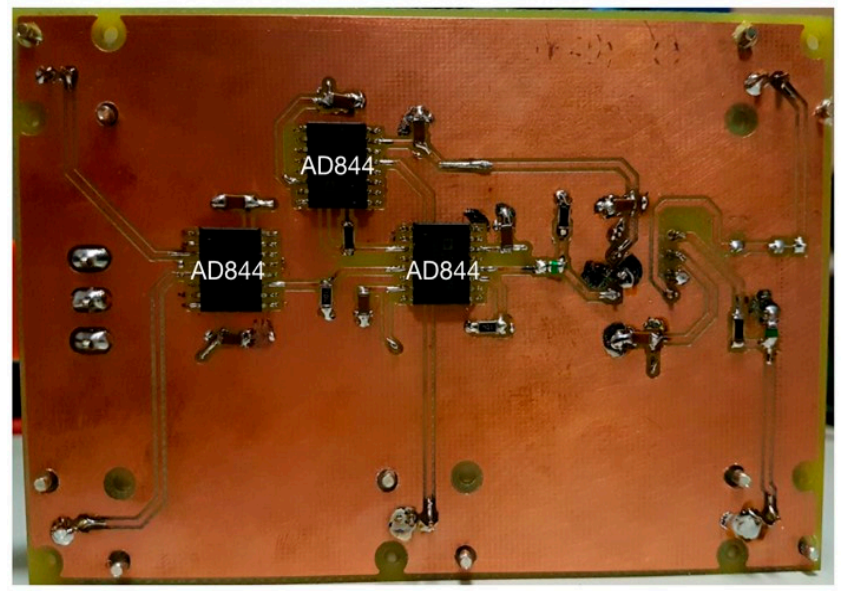

(a)

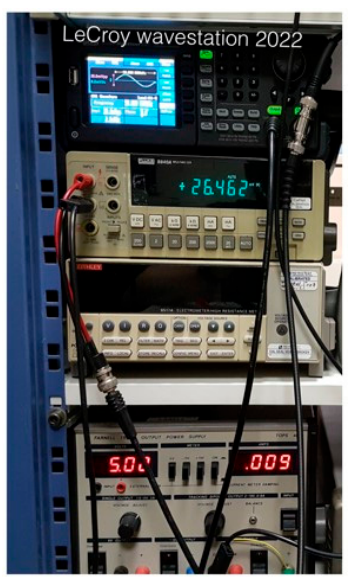

(b)

Figure 3. (a) Bottom view of the implemented board-level CM-TIA prototype. (b) Experimental setup.
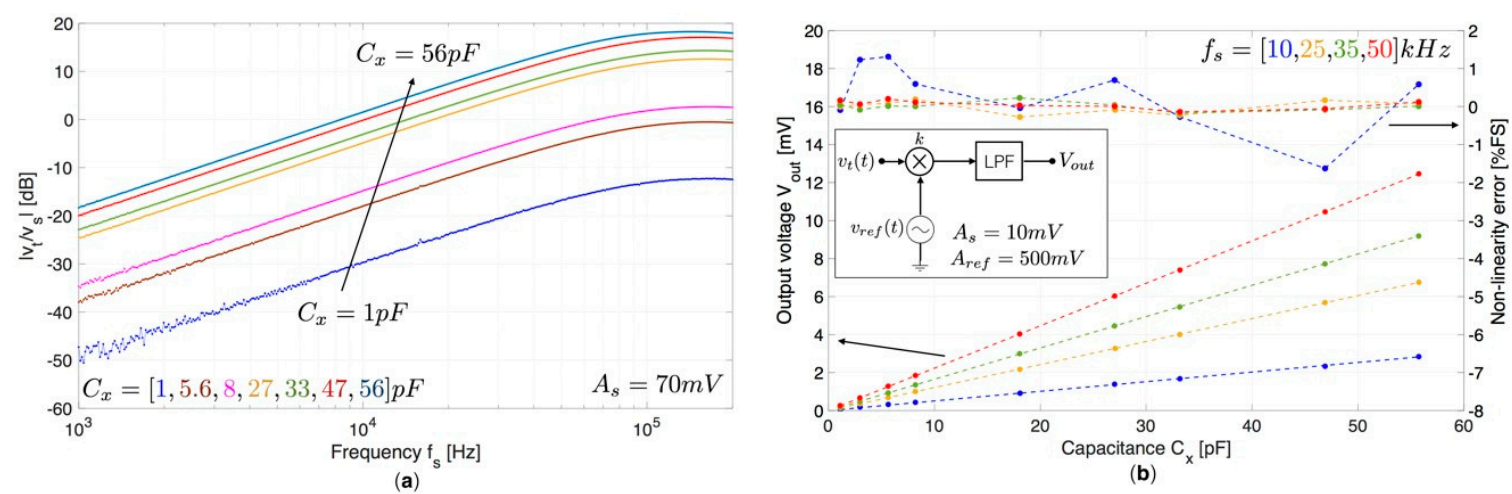

Figure 4. (a) Magnitude of the ratio $v_{t} / v_{s}$ as a function of the frequency $f_{s}$ obtained for different values of reference capacitance $C_{x}$. (b) Measured output voltage $V_{\text {out }}$ as a function of the reference capacitance $C_{x}$ for different values of $f_{s}$. Raw data are represented with dots while dotted-lines represent fittings with a first-order polynomial. The calculated non-linearity error as a function of $C_{x}$ is also reported considering as full-scale FS the maximum output voltage $V_{\text {out }}$ obtained at each operating frequency.

A good agreement with the theoretical behavior is obtained, showing a linear trend for frequencies up to $80 \mathrm{kHz}$, exhibiting a lower bandwidth with respect to the proposed optimized integrated version, as reported in Figure 4a. A synchronous demodulation has been implemented to provide a DC output voltage and improve the signal-to-noise ratio by using an analog multiplier (AD835) and a low-pass filter as reported in the inset of Figure $4 \mathrm{~b}$. The sinusoidal reference voltage $v_{\text {ref }}(\mathrm{t})$ has a peak amplitude $A_{\text {ref }}=0.5 \mathrm{~V}$ and a nominal phase shift $\phi=-90$ deg with respect to the excitation signal $v_{\mathrm{s}}(\mathrm{t})$ whose peak amplitude is $A_{\mathrm{s}}=10 \mathrm{mV}$. The $\mathrm{DC}$ output signal is equal to:

$$
V_{\text {out }}=0.5 \mathrm{k}^{-1} \mathrm{G} A_{\mathrm{s}} A_{\text {ref }} 2 \pi f_{\mathrm{s}} C_{\mathrm{x}} \cos (\phi+90)
$$

where $\mathrm{k}=1.05 \mathrm{~V}$ is the multiplier gain factor. Figure $4 \mathrm{~b}$ shows the measured output voltage $V_{\text {out }}$ as a function of the reference capacitance $C_{x}$ obtained at different frequency values $f_{\mathrm{s}}$ of $10,25,35,50 \mathrm{kHz}$. Experimental results are in good agreement with the expected values, showing a good linearity within the measurement range. The obtained maximum sensitivity is about $223 \mathrm{mV} / \mathrm{nF}$ for $f_{\mathrm{s}}=50 \mathrm{kHz}$ with an estimated resolution calculated at $3 \sigma_{V_{\text {out }}}=7.68 \mu \mathrm{V}$ of about $34.5 \mathrm{fF}$.

\section{Conclusions}

A current-mode transimpedance amplifier based on second generation current conveyors able to measure capacitive variation with low sensing signal amplitude has been presented.

The integration of CCII promotes the design of low-power integrated circuits, overcoming restraints typically provided by circuits based on operational amplifiers, ensuring a larger frequency 
bandwidth and a good capability to work at a low supply voltage levels with a wide dynamic range. The proposed electronic interface performs a capacitance-to-voltage conversion using 3 CCIIs and 3 resistors exploiting a synchronous-demodulation technique to improve the overall sensitivity and resolution of the system. Experimental results obtained with a board-level prototype have led to a sensitivity of about $223 \mathrm{mV} / \mathrm{nF}$ for $f_{\mathrm{s}}=50 \mathrm{kHz}$ with an estimated resolution calculated at $3 \sigma_{V_{\text {out }}}=7.68$ $\mu \mathrm{V}$ of about $34.5 \mathrm{fF}$. The obtained values of sensitivity and resolution show that the proposed $\mathrm{CM}-$ TIA is suitable for capacitive measurement of comb-finger parallel-plate MEMS sensors.

Conflicts of Interest: The authors declare no conflict of interest.

\section{References}

1. Xia, F.; Campi, F.; Bahreyni, B. Tri-mode capacitive proximity detection towards improved safety in industrial robotics. IEEE Sens. J. 2018, 18, 5058-5066, doi:10.1109/JSEN.2018.2832637.

2. Lee, J.C.; Yang, S.H. Development of nanopositioning mechanism with real-time compensation algorithm to improve the positional accuracy of a linear stage. Precis. Eng. 2017, 50, 328-336, doi:10.1016/j.precisioneng.2017.06.006.

3. Molinnus, D.; Muschallik, L.; Gonzales, L.O.; Bongaerts, J.; Wagner, T.; Selmer, T.; Siegert, P.; Keusgen, M.; Schöning, M.J. Development and characterization of a field-effect biosensor for the detection of acetoin. Biosens. Bioelectron. 2018, 115, 1-6, doi:10.1016/j.bios.2018.05.023.

4. Zhao, Y.; Yang, B.; Liu, J. Effect of interdigital electrode gap on the performance of $\mathrm{SnO}_{2}$-modified $\mathrm{MoS}_{2}$ capacitive humidity sensor. Sens. Actuator B Chem. 2018, 271, 256-263, doi:10.1016/j.snb.2018.05.084.

5. Ren, Y.; Pan, M.; Chen, D.; Tian, W. An Electromagnetic/Capacitive Composite Sensor for Testing of Thermal Barrier Coatings. Sensors 2018, 18, 1630, doi:10.3390/s18051630.

6. Xue, N.; Gao, G.; Sun, J.; Liu, C.; Li, T.; Chi, C. Systematic study and experiment of a flexible pressure and tactile sensing array for wearable devices applications. J. Micromech. Microeng. 2018, 28, 075019, doi:10.1088/1361-6439/aabc88.

7. Aydemir, A.; Terzioglu, Y.; Akin, T. A new design and a fabrication approach to realize a high performance three axes capacitive MEMS accelerometer. Sens. Actuator A Phys. 2016, 244, 324-333, doi:10.1016/j.sna.2016.04.007.

8. Nastro, A.; Ferrari, M.; Russo, A.-L.; Ardito, R.; Ferrari, V. MEMS force sensor with DDS-based position feedback and tunable sensitivity. In Proceedings of the IEEE Sensors Conference, Glasgow, Scotland, 29 October-1 November 2017; doi:10.1109/ICSENS.2017.8234151.

9. Nastro, A.; Ferrari, M.; Russo, A.-L.; Ardito, R.; Ferrari, V. Servo-Assisted Position-Feedback MEMS Force Sensor with Tunable Sensitivity and Sub-Nanonewton Range. In Proceedings of the Eurosensors17 Conference, Paris, France, 3-6 September 2017; doi: 10.3390/proceedings1040383.

10. Cerini, F.; Ferrari, M.; Ferrari, V.; Russo, A.; Urquia, M.A.; Ardito, R.; de Masi, B. Electro-Mechanical Modelling and Experimental Characterization of a High-Aspect-Ratio Electrostatic-Capacitive MEMS Device. Sens. Actuators A Phys. 2017, 266, 219-231, doi:10.1016/j.sna.2017.07.048.

11. De Marcellis, A.; Di Carlo, C.; Ferri, G.; Stornelli, V. A CCII-based wide frequency range square waveform generator. Int. J. Circuit Theory Appl. 2013, 41, 1-13, doi:10.1002/cta.781.

12. López-Martín, A.J.; Ramirez-Angulo, J.; Carvajal, R.G. Highly accurate CMOS second generation current conveyor and transconductor. In Proceedings of the IEEE EUROCON, Salamanca, Spain, 8-11 September 2015; doi:10.1109/EUROCON.2015.7313765.

13. Reda, A.; Ibrahim, M.F.; Farag, F. Input-output rail-to-rail CMOS CCII for low voltage-low power applications. Microelectron. J. 2016, 48, 60-75, doi:10.1016/j.mejo.2015.12.001.

14. De Marcellis, A.; Ferri, G.; Mantenuto, P. A CCII-based non-inverting Schmitt trigger and its application as astable multivibrator for capacitive sensor interfacing. Int. J. Circuit Theory Appl. 2017, 45, 1060-1076, doi:10.1002/cta.2268.

15. De Marcellis, A.; Palange, E.; Liberatore, N.; Mengali, S. Low-cost portable 1MHz lock-in amplifier for fast measurements of pulsed signals in sensing applications. IEEE Sens. Lett. 2017, 1, 1-4, doi:10.1109/LSENS.2017.2713449. 